\title{
Patch Antenna for 2.7GHz \& 5.6GHz for CMMB
}

\author{
Muhammad Noman ${ }^{1}$, Muhammad Usman ${ }^{2}$ and Muhammad Zeeshan ${ }^{3}$ \\ ${ }^{1,3}$ Department of Electrical Engineering, COMSATS Institute of Information \\ Technology, Islamabad, Pakistan \\ ${ }^{2}$ Department of Electrical Engineering, Center of Advanced Studies in Engineering \\ (CASE), Islamabad, Pakistan \\ 'noman049@gmail.com, ${ }^{2}$ muhammadusman92@ymail.com, \\ 33ohammadzeeshan98@yahoo.com
}

\begin{abstract}
The advantages of micro strip patch antennas have made them a perfect candidate for use in the local area network (WLAN) applications. This paper represents the new design of micro strip patch antenna for China Multimedia Mobile Broadcast (CMMB). In the designed micro strip patch antenna, we applied different techniques to achieve the resonant frequency of $2.7 \mathrm{GHz}$ with the bandwidth of $300 \mathrm{MHz}$. Unique slots and conventional slits are introduced in such a way that not only enhanced the bandwidth but also provided us with the fruitful result of $-74.78 \mathrm{~dB}$ return loss. Its minor non-symmetry led to dual frequency i.e. $5.60 \mathrm{GHz}$ with the bandwidth of $600 \mathrm{MHz}$ and return loss of $-15.41 \mathrm{~dB}$. Further the details regarding to the dimensions of the patch, ground and simulations are elaborated.
\end{abstract}

Keywords: Micro strip patch antenna, China Multimedia Mobile Broadcast (CMMB), resonant frequency, slits, return loss

\section{Introduction}

In the previous century the communication started evolution with the progress in the industry. First the communication was wired which was expensive and tiresome to use, so the world wanted to move on wireless communication to avoid the wired structures which limit mobility of the user.

With the fast development in the technology and wireless communication devices, the field requires the low profile, less expensive and compact antennas. Micro strip patch antennas are the main focus of the most researchers due to its low fabrication cost and compactness ability. As we know that antenna is a conducting device which is used for radiating and receiving radio waves $[1,2]$. Few of the many types of antennas are wire antenna, aperture antenna, micro strip patch antenna, array antenna, reflector antenna etc. Micro strip patch antenna (also known as printed antenna) [3, 4] is the core focus of our research because it is easy to manage, fabricate and analyze [5-7]. The construction of micro strip patch antenna includes a metallic patch which is printed on a substrate of different materials mounted on a ground [8]. Moreover the shape and geometry of the patch can vary from model to model depending upon the requirement. Our designed antenna not only operates on $2.7 \mathrm{GHz}$ resonant frequency with $300 \mathrm{MHz}$ bandwidth but it is also operatable on $5.60 \mathrm{GHz}$ with $600 \mathrm{MHz}$ bandwidth. Insertion of multiple rectangular slots of different dimensions following a chebyschev distribution in a rectangular patch at about the centre of patch as shown in Figure 1. The position of the slots is managed by the precise analysis of magnitude surface charge density. The 
first technique in our design was the partial grounding and the addition of slit slots in it to enhanced the bandwidth. Secondly two small circles of radius $=0.23 \mathrm{~mm}$ are introduced in the area of the junction of feed and patch for the impedance matching which resulted the return loss of $-74.78 \mathrm{~dB}$ as shown in Figure 2. Keeping in mind the idea of giving a proper direction to the flowing current, an inverted V slot using poly line is constructed above the chebyshev distribution. This slot was introduced to give the direction to the current towards maximum surface charge density area and make a radiating edge there as shown in Figure 3. The edge truncation led the antenna to be circularly polarized.

A sample antenna was analyzed, simulated, fabricated and tested. Several results were presented and discussed. The said antenna is applicable for CMMB, Wimax, MMDS (Multi channel Multipoint Distribution System.

\section{Source of Idea and its Implementation}

The source of idea of the central slots and the pin type circles on the patch near the junction of patch and feed line was taken by the paper referred as [2] and [3] respectively.

First idea was taken from [2]". In the source of idea the slots were horizontally (right) directed, where as in our antenna it is north or vertical directed.

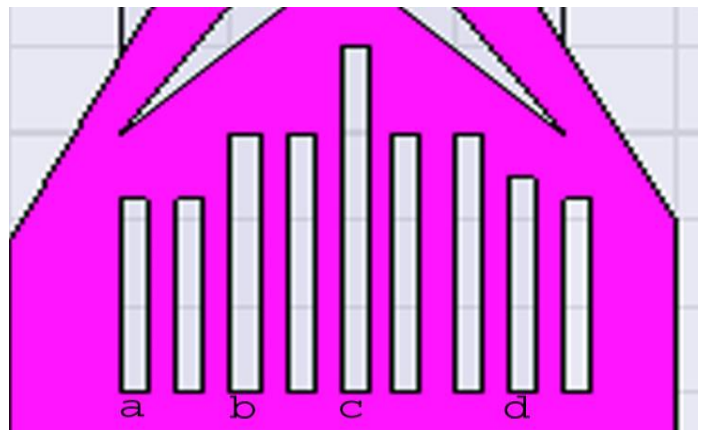

Figure 1(a). North or Vertically Directed Slots of Antenna

\begin{tabular}{|c|c|}
\hline Slot name & $\begin{array}{c}\text { Dimensions (L x W) } \\
\text { in } \mathrm{mm} .\end{array}$ \\
\hline a & $4.5 \times 0.5$ \\
\hline b & $6 \times 0.5$ \\
\hline c & $8 \times 0.5$ \\
\hline$d$ & $5 \times 0.5$ \\
\hline
\end{tabular}

Figure 1(b). Dimensions for Slots of Figure 1(a)

Second idea was taken from [3]". Initially the idea was to implement the preceding mentioned technique the way it was implemented but in our case the results were getting spoiled and we had to trade off with the return loss, so the idea was given a new shape.

Rather drilling in the substrate we made small hole in the united patch on a suitable position and the dimension was $\mathrm{r}=0.23 \mathrm{~mm}$. This gave a drastic change in the return loss, and by introducing another hole on a suitable location the return loss got the figure of $74.78 \mathrm{~dB}$. 


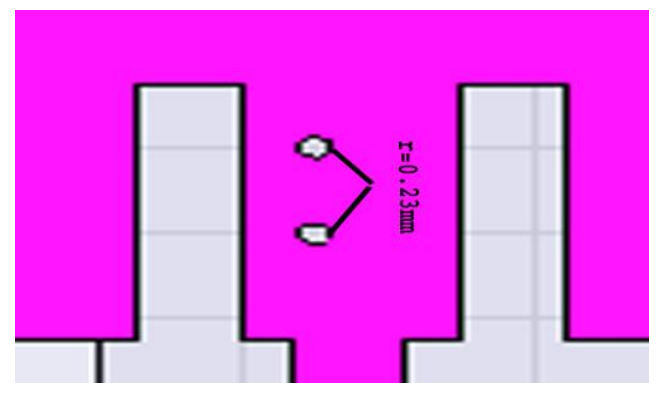

Figure 2. Radius of both Holes $=0.23 \mathrm{~mm}$

\section{Antenna Structures and Results}

The fundamental three layers of the constructed antenna comprises of ground, substrate and the patch. The dimensions for ground and substrate are $40 * 40(\mathrm{~mm})$. Material assigned to the substrate is FR4_eproxy with relative permittivity=4.4, dielectric loss tangent- 0.02 and lande $\mathrm{G}$ Factor=2. The upper layer is the patch consists of rectangle of $19 * 25 \mathrm{~mm}$ joined with the triangle using poly lines. In the rectangular patch 9 rectangular slots are inserted which increases the beamwidth and resonances. Further in triangular patch an inverted V shaped slot is inserted with the help of poly lines on a specific location determined by surface charge density from the field overlays. This made a radiating edge on the pointed sides of the antenna resulting in an increased radiation. The two rectangular slots of dimensions $12 * 2 \mathrm{~mm}$ and $18 * 2 \mathrm{~mm}$ in addition with poly line slots were united and inserted in the patch to give direction to the current towards the radiating edge made by the $\mathrm{V}$ shaped slot on top of the patch.

In the initial phase of construction the feed line was made with the dimensions of $12 * 2.9 \mathrm{~mm}$ but later on by curtailing the width of feed line with $0.2 \mathrm{~mm}$ we were getting increment in return loss indicating the impedance to be matched with that of patch. And finally the optimal width we got for feed line was $1.5 \mathrm{~mm}$ resulting the return loss of $74.78 \mathrm{~dB}$ which is an evidence of impedance matching. Since we have two references for SMA female connector i-e 50ohms and $75 \mathrm{ohms}$ impedance, in our case we will be using 75ohm SMA female connector.

During its constructions many antennas with the similar geometry were obtained and many shapes were introduced replacing the presently used shapes. One of the technique used while its construction was to replace the chebyshev distribution with a small hole of radius $=0.28 \mathrm{~mm}$ which takes the return loss to $-60 \mathrm{~dB}$ as shown in Figure 3 .

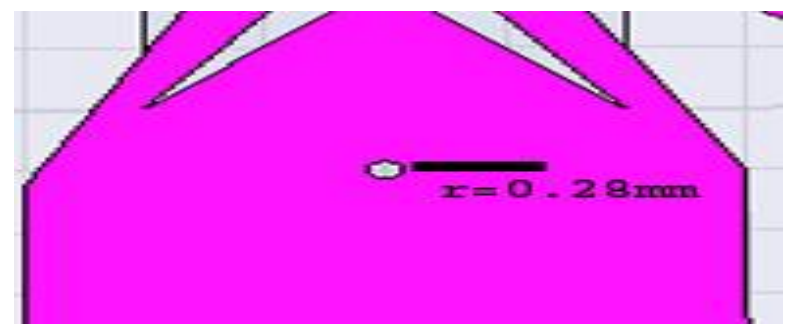

\section{Figure 3. Chebyshev Replaced by Hole of Radius $=0.28 \mathrm{~mm}$}

The partial grounding technique gave us a very fruit full result in case of $5.60 \mathrm{GHz}$ frequency band. It increased the bandwidth to $600 \mathrm{MHz}$ which made this antenna a wide band because of its characteristics. The two slits and one slot were made to adjust the 
dip on $5.60 \mathrm{GHz}$ and bandwidth adjustment. The slits and slots are made on optimal positions participating constructively in the results of both the operating frequencies of antenna.

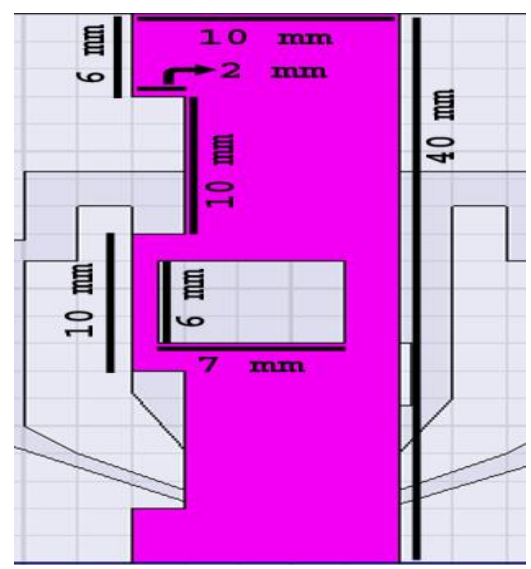

Figure 4. Partial Grounding and Slits Slots on Ground

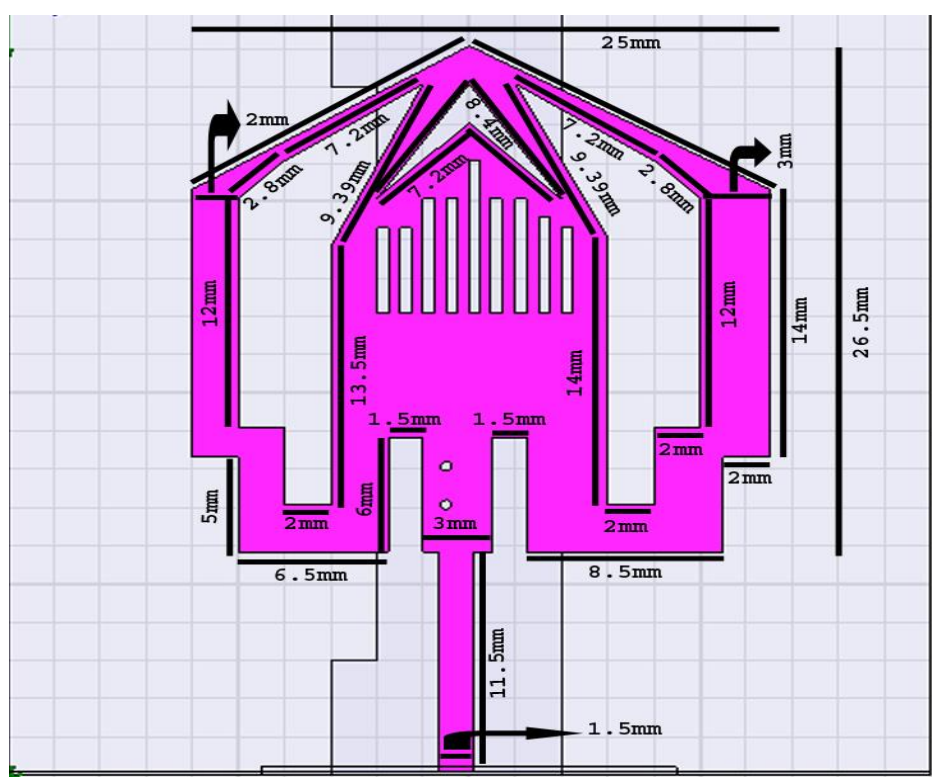

Figure 5. The New Antenna Structure

\section{GAIN and VSWR}

The gain for the constructed antenna is $-1 \mathrm{~dB}$ and VSWR (Voltage Standing Wave Ratio) for $2.7 \mathrm{GHz}$ and $5.60 \mathrm{GHz}$ respectively are 1.01 and 1.41 . The diagrams for VSWR and Gain are represented in Figure 5 and Figure 6.

It is a common observation that as many slits and slots we are going to introduce the less is the gain, but in this case a number of slits and slots made the gain -1 . 


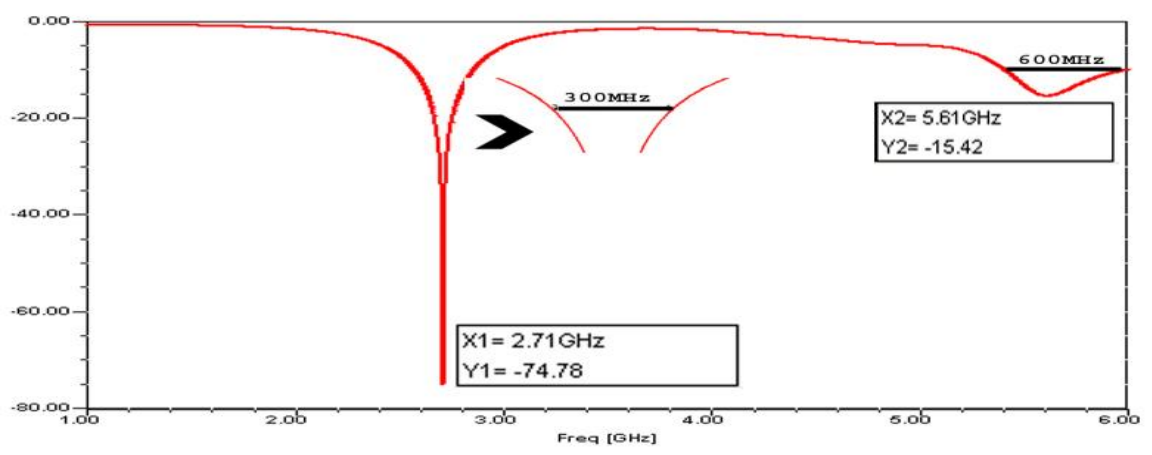

Figure 6. Return Loss and Bandwidth on Both Resonant Frequencies

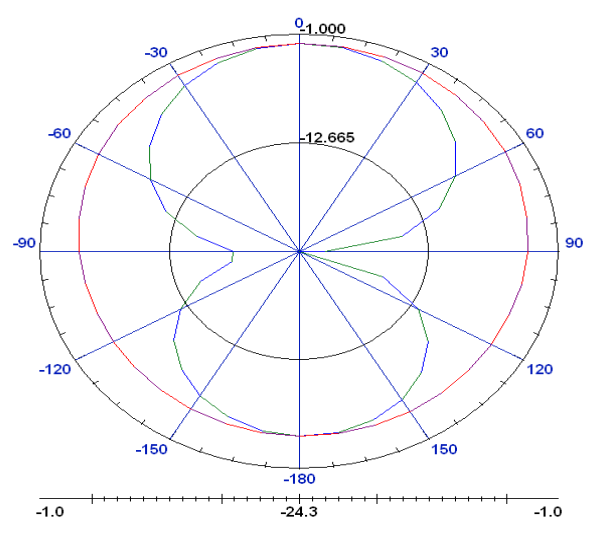

Figure 7. -1 Gain

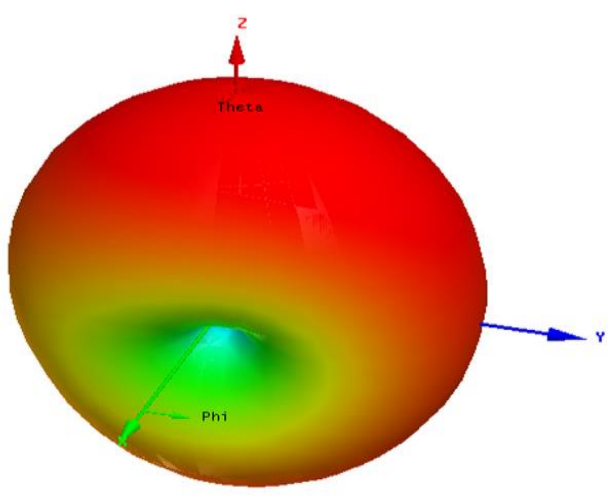

Figure 8. 3D Radiation Pattern

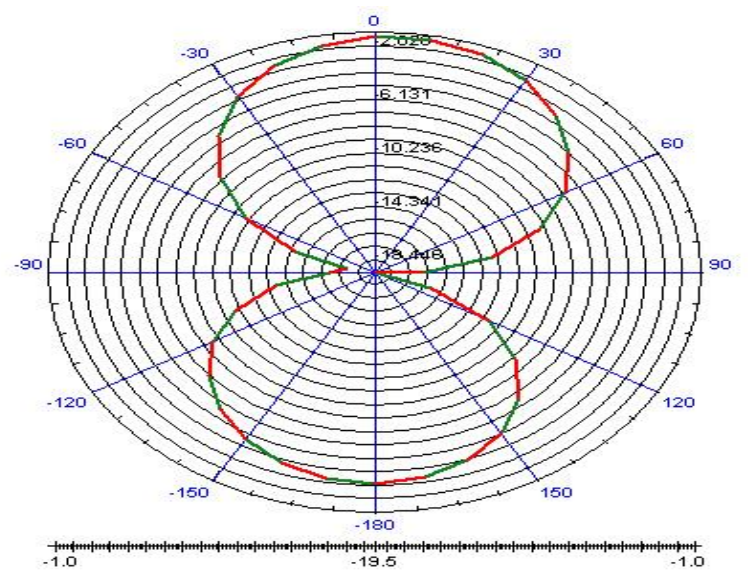

Figure 9. Radiation Pattern in H-plane

\section{Conclusion}

The paper illustrates a new vision of geometry which produces fruitful results when implemented successfully. The purpose of the work is to design a small size, less power consumed and low cost antenna that can be used for wideband communication applications. 
It describes the method of obtaining dual band by creating unsymmetrical side, holes of correct dimension and correct position for impedence matching that led to $-74.78 \mathrm{~dB}$ return loss. Wimax and China Multimedia Mobile Broadcasting are the major applications for the constructed antenna.

\section{References}

[1] P. J. Soh, M. K. A. Rahim, A. Asrokin and M. Z. A. Abdul Aziz, "Comparative radiation performance of different feeding techniques for a microstrip patch antenna", Proceedings of the Asia-Pacific Conference on Applied Electromagnetics, Johor Bahru, Johor, Malaysia, (2005) December 20-21.

[2] J. Costantine, K. Y. Kabalan, A. El Hajj and C. G. Christodoulou, "New multi band design for a microstrip patch antenna", Proceedings of the second European conference on Antennas and Propagation, EICC, Edinburgh, UK, (2007) November 16.

[3] J.-S. Row, "A simple impedance-matching technique for patch antennas fed by coplanar microstrip line", IEEE Transactions on Antennas and Propagation, vol. 53, no. 10, (2005) October, pp. 3389-3391.

[4] V. G. Veselago, "The electrodynamics of substances with simultaneously negative values of IMG align= ABSMIDDLE alt= $\epsilon$ eps/IMG and $\mu$, Sov. Phys. Uspekhi”, vol. 10, no. 4, (1968), pp. 509-514.

[5] C. A. Balanis, “Antenna Theory and Design”, John Wiley \& Sons Publishers, New York, (1997).

[6] W. L. Stutzman and G. A. Thiele, “Antenna Theory and design”, John Wiley \& Sons Publishers, New York (1998).

[7] J. B. Pendry, "Magnetism from conductors and enhanced nonlinear phenomena", IEEE Trans. Micro Tech., vol. 47, no. 11, (1999), pp. 2075-2081.

[8] D. R. Smith, W. J. Padilla, D. C. Vier, Sc C. Nemat-Nasser and S. Schultz, "Composite medium with simultaneously negative permeability and permittivity”, Physical review letters, vol. 84, no. 18, (2000).

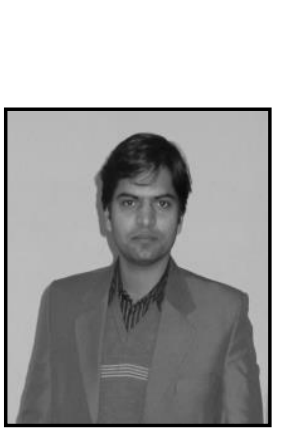

\section{Authors}

Muhammad Noman received his bachelor degree in Electrical (Telecommunication) Engineering from COMSATS Institute of Information Technology, Islamabad in 2011.

Ever science then he is working at various engineering positions with an IT and Engineering Organization in Islamabad, Pakistan. He is a designated reviewer and technical program committee member at various international journal and conference publishers. His research interests includes antenna designing, image processing techniques, wireless and mobile communications, 3G, 4G and NGNs. Proceedings.

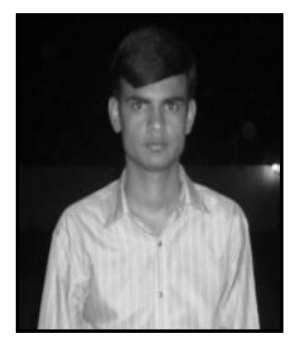

Muhammad Usman is presently doing bachelor degree in Electrical (Telecommunication) Engineering from Center for Advanced Studies in Engineering (CASE), Islamabad. His research interests includes antenna designing, image processing techniques, wireless and mobile communications.

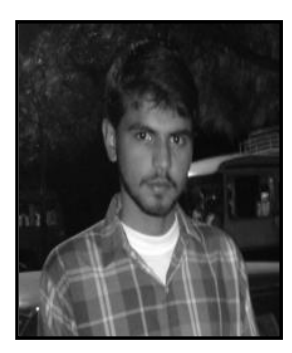

Muhammad Zeeshan is presently doing bachelor degree in Electrical (Power) Engineering from COMSATS Institute of Information Technology, Islamabad. His research interests includes antenna designing, power electronics, circuit analysis, wireless and mobile communications 